\title{
Pseudomonas aeruginosa outer membrane proteins: Profile analysis and immunogenicity in relation to the antibiotic resistance
}

\author{
Mona I. El-Enbaawy ${ }^{1}$, Ansam M.Salam ${ }^{2}$ \\ ${ }^{1}$ Department of Microbiology, Faculty of Veterinary Medicine Cairo University, Cairo, Egypt \\ ${ }^{2}$ Department of Immunology, Animal Health Institute, Dokki, Giza, Egypt
}

To analyze the major outer membrane proteins (OMPs) of the sensitive or resistant Pseudomonas aeruginosa strains, the OMPs were separated from the cellular elements by sarcosyl extraction method. OMPs profiles were conducted by SDSpolyacrylamide gel electrophoresis. Amoxicillin clavulanic acid (AMC) sensitive $P$. aeruginosa serotype $\mathrm{K}$ showed four protein bands; 35.713, 31.159, 26.107 and 22.869 KD. While AMC sensitive P. aeruginosa serotype $H$ showed three bands of 35.713, 27.164 and 23.174 KD. Whereas AMC resistant $P$. aeruginosa serotype G, that was positive for the $b^{\prime} a_{\mathrm{TEM}}$ gene by the PCR, modified its protein pattern. It has five protein bands of $52.142,38.525,30.690,27.164$ and 22.569 KD. These findings suggested that $b_{1} a_{\text {TEM }}$ gene and the outer membrane protein barrier are contributed to the resistance to amoxicillin clavulanic acid in $P$. aeruginosa. To determine a possible relationship between the resistance of $P$. aeruginosa and the production of antibodies against its outer membrane protein, antibodies against OMPs of AMC sensitive and resistant $P$. aeruginosa strains were prepared in mice and evaluated by ELISA. Our results showed that there was no association between immunogenicity of the outer membrane proteins and resistance of $P$. aeruginosa to antibiotics.

Pseudomonas aeruginosa is a clinically significant pathogen that exhibits intrinsic resistance to various antimicrobial agents (Quinn, 2002). The B-lactamase inhibitor; amoxycillin clavulanic acid (trade name; coamoxyclav or synulox or augmentin) is indicated for treatment of both Gram positive and Gram negative bacterial infection in both human and veterinary practice (Ball et al., 1980). Little is recorded about amoxycillin clavulanic acid susceptibility of $P$. aeruginosa in animals.

Many reports have demonstrated that the intrinsic resistance of $P$. aeruginosa to betalactams is due to the interplay among multiple resistance mechanisms (Li et al., 2000 and Putman et al., 2000). Among which; plasmidencoded TEM-type $\beta$-lactamase (bla $a_{\mathrm{TEM}}$ gene) (Kaye et al., 2004) and the inhibition of drug entry into the cell (Putman et al., 2000).

Attention has been addressed to the outer membrane proteins of $P$. aeruginosa and their consideration as a penetration barrier responsible for some antibiotic resistance $P$. aeruginosa (Cullmann et al., 1986). The first aim of this study was to analyze the major OMPs of the sensitive or resistant $P$. aeruginosa strains for a comparison between them by SDS- polyacrylamide gel electrophoresis.

$P$. aeruginosa OMPs are valuable immunogens (Gilleland et al., 1984). Previous studies have been performed to evaluate immunogenicity of the outer membrane proteins of $P$. aeruginosa in human and animals (Alexander and Fisher, 1974; Gilleland et al., and Jang et al., 1999).

A trial was performed to determine a possible relationship between the resistance of $P$. aeruginosa and the production of antibodies against its outer membrane protein.

\section{Material and Methods}

Organisms. Six isolates of $P$. aeruginosa from samples of chicken origin were included in this work. The six strains belong to serotype F, L G, $\mathrm{K}, \mathrm{H}$ and $\mathrm{F}$ as shown in (Table 1 ).

Antibiotic susceptibility. The antibiotic susceptibilities of the $P$. aeruginosa isolates were analyzed by the disk diffusion method using Mueller-Hinton broth and agar (Oxoid). Antibiotic discs (Oxoid, Ltd., London, England) were used and interpretation was done as described by the National committee for clinical laboratory standards (1993). The following antibiotics were tested: amoxicillin-clavulanic

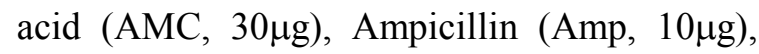
ceftriaxone $(\mathrm{CRO}, 30 \mu \mathrm{g})$, neomycin $(\mathrm{N}, 30 \mathrm{ug})$, 
streptomycin (S, 10ug) and norfloxacillin (NOR, $10 \mu \mathrm{g})$.

Detection of $\boldsymbol{b l a}$ TEM gene: PCR amplification of the bla $a_{T E M}$, gene was carried out for screening purposes, as described (Belaaouaj et al., 1994). $P$. aeruginosa cells were lysed by heating and cellular debris was removed by centrifugation. The supernatant was used as the templates for the following PCR. Primers MATEMF (5'ATGA GTATTCAACATT TTCGTGC-3') and MATEMR (5'CCAATGCTTAATCAGTGAGGCAC C-3') were used. The PCR program was as follows: $95^{\circ} \mathrm{C}$ for $5 \mathrm{~min}$ and then 35 cycles of $94^{\circ} \mathrm{C}$ for $60 \mathrm{~s}, 58^{\circ} \mathrm{C}$ for $60 \mathrm{~s}$, and $72^{\circ} \mathrm{C}$ for 60 $\mathrm{s}$ followed by $72^{\circ} \mathrm{C}$ for $10 \mathrm{~mm}$. The amplified product was visualized by standard gel electrophoresis of $10 \mu \mathrm{l}$ of the PCR mixture on $1.5 \%$ agarose gel stained with $0.5 \mu \mathrm{g} / \mathrm{ml}$ ethidium bromide. The amplicon size was evaluated by comparison band with the sizes of the fragments of Hae III molecular weight marker (Invitrogen, USA).

Outer membrane proteins preparation. The outer membrane proteins of AMC resistant and sensitive $P$. aeruginosa strains were prepared as described by (Caballero et al., 2001). Basically, bacteria were grown at $37^{\circ} \mathrm{C}$ in nutrient agar (Oxoid); the cells were harvested and broken by sonication in an ice bath $(20 \mathrm{kHz}$ for 2 minutes, 5 cycles). Whole cells were eliminated by centrifugation at 5000 $g$ for 20 minutes. The outer membrane proteins were separated from the other cellular components by sarcosyl extraction (N-lauroylsarcosine $\mathrm{Na} 2 \%$ concentration) at room temperature for 30 minutes followed by centrifugation $\left(38000 \mathrm{~g}\right.$ for 1 hour at $\left.4^{\circ} \mathrm{C}\right)$. The pellets with outer membranes were resuspended in $50 \mathrm{mM}$ Tris-HCL (PH 6.8) and frozen at $70^{\circ} \mathrm{C}$ until used. The total protein concentration of the preparation was calculated by the method of (Lowry et al., 1951).

OMPs analysis by SDS-polyacrylamide gel electrophoresis (SDS-PAGE). The OMPs were analyzed using vertical electrophoresis in $10 \%$ (wt/vol) SDS-polyacrylamide gel as described by (Laemmli, 1970) with a Protean II slab electrophoresis system (Bio-Rad Laboratories, Richmond, Calif. USA). Protein fractions were solubilized in sample buffer, heated at $95^{\circ} \mathrm{C}$ for $5 \mathrm{~min}$. The OMPs were loaded in amounts of approximately $35 \mathrm{mg}$ and separated by sodium dodecyl sulfate-polyacrylamide gel electrophoresis (SDS-PAGE) with 10\% (wt/vol) acrylamide and $0.1 \%$ (wt/vol) piperazine diacrylyl in the running gel at a constant current of $20 \mathrm{Am}$. The gel was stained with Coomassie brilliant blue R-250 (Bio-Rad). Quantitation of the stained OMPs bands was done by using scanning spectrophotometry at absorbance of 605 nm(Hames and Rickwood,1987).

Production of antibodies against OMPs in mice. Antibodies against OMPs of sensitive and resistant strains of $P$. aeruginosa strains were prepared as described by (Muthukumar and Muthukkaruppan, 1993). Four groups of 10 mice, were immunized with the OMPs antigens of $P$. arugenosa. Each mouse was injected with $50 \mathrm{ug} / 0.2 \mathrm{ml}$ of the indicated antigen subcutaneously $(\mathrm{S} / \mathrm{C})$ twice at 15 day intervals. The first, the second and the third groups were immunized with OMPs antigen of $P$. aruginosa AMC sensitive serotype $\mathrm{K}$, AMC sensitive serotype $\mathrm{H}$ and AMC resistant serotype $\mathrm{G}$, respectively. The fourth group served as control non-injected group. Fifteen days after the last injection, sera were collected from each group of mice and stored at -20 till used.

Detection of the antibody level (Leitner et al., 1990). The humoral immune response was assessed, $15 \mathrm{~d}$ after the $2 \mathrm{nd}$ immunization. The indicated OMPs antigens $(20 \mu \mathrm{g} / \mathrm{ml})$ in carbonate buffer was used to coat 96-well microtiter plates (Nunc Immunoplates), which were then incubated at $4^{\circ} \mathrm{C}$ overnight. Following antigen coating, ELISA plates were washed and blocked with $3 \%$ bovine serum albumin in PBS for $1 \mathrm{~h}$ prior to addition of the serum. Serum was diluted in PBS containing $0.3 \%$ bovine serum albumin and $0.05 \%$ Tween 20. Following serial dilution, diluted sera were incubated in the wells for $2 \mathrm{~h}$ at $37^{\circ} \mathrm{C}$. Wells were washed with PBS containing $0.05 \%$ Tween $20,100 \mu 1$ of the appropriate goat antimice IgG conjugate was added for $2 \mathrm{~h}$ at $37^{\circ} \mathrm{C}$. Wells were then washed, and bound conjugate was detected by using ABTS peroxidase substrate. Reactions were stopped with $20 \mu 1$ of $2.5 \mathrm{M} \mathrm{H}_{2} \mathrm{SO}_{4}$ and optical densities (ODs) for the tested and control sera were read at $405 \mathrm{~nm}$.

\section{Results}

Characterization of clinical strains. It was illustrated in (Table 1), by disk diffusion method, that three strains (serotypes; F, L and G) were resistant to amoxicillin clavulanic acid (zone diameter $\leq 13 \mathrm{~mm}$ ) and other antibiotics; ampicillin, ceftriaxone, neomycin and streptomycin. Three strains (serotypes; K, H and F) were sensitive to amoxicillin clavulanic acid (zone diameter $\geq 17 \mathrm{~mm}$ ). The bla $_{\mathrm{TEM}}$ gene was 


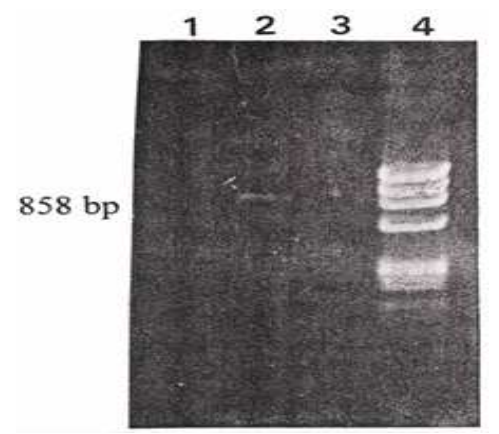

Fig. (1): The amplification of 858 bp fragment of $P$. aeruginosa bla $a_{\mathrm{TEM}}$ gene positive, Lane (2). $P$. aeruginosa negative for $b a_{\mathrm{TEM}}$ gene Lane (1 and 3). Hae III molecular weight marker (Lane). recovered from only one of three $P$. aeruginosa isolates (serotype G). PCR amplification product of $858 \mathrm{bp}$ fragment of $b_{\text {bla }}$ gene was shown in Fig. (1).

The SDS-PAGE profile of the outer membrane proteins of AMC sensitive and resistant strains of $P$. aeruginosa. As shown in (Fig 2), not all the visible Coomssie blue stained bands were quantitated by the scanning spectrophotometer at absorbance of $605 \mathrm{~nm}$. AMC sensitive $P$. aeruginosa serotype $\mathrm{K}$ induced four bands; 35.713, $31.159,26.107$ and 22.869 KD. While AMC sensitive $P$. aeruginosa serotype $\mathrm{H}$ induced three bands; 35.713, 27.164 and 23.174 KD. Whereas AMC resistant $P$. aeruginosa serotype $\mathrm{G}$ and positive for $b l a_{\mathrm{TEM}}$ gene modified the protein pattern of its OMPs. It has five bands of 52.142, 38.525, 30.690, 27.164 and 22.569 KD (Table 2).

The immunigenic differences afforded by OMPs of AMC sensitive or resistant bla $_{\text {TEM }}$ gene positive $P$. aeruginosa strains. It was clear from Table (3) that there was a significant increase in antibody levels against OMPs of sensitive and resistant $P$. aeruginosa but without differences in the OD values within mice groups injected with the OMPs antigens, in comparison with the control group. It is interesting to note that there was no relation

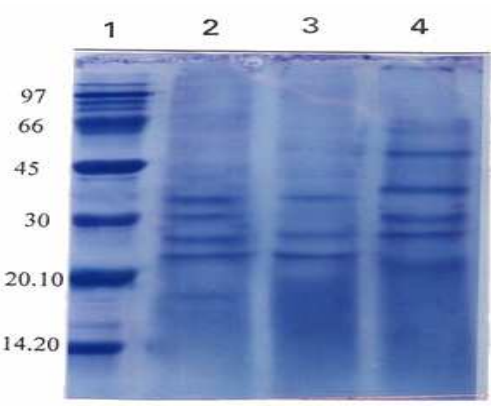

Fig. (2): Outer membrane proteins profile analysis for sensitive and resistance strains of $P$. aeruginosa that was separated by SDS-PAGE electrophoresis. Lane (1) is the molecular weight marker in KD. Lane (2) is the AMC sensitive $P$. aeruginosa serotype $K$. Lane (3) is the AMC sensitive $P$. aeruginosa serotype $H$. Lane (4) is the AMC resistant $P$. aeruginosa serotype $G$ and positive for $b^{\prime a} a_{\mathrm{TEM}}$ gene. Arrows indicate Outer membrane protein bands, which were subjected to scanning spectrophotometric analyses.

between numbers of protein bands of the OMPs of $P$. aeruginosa detected by SDSPAGE and the levels of anti-outer membrane protein antibodies detected by ELISA.

\section{Discussion}

Pseudomonas aeruginosa is known for its ability to develop resistance to many antibiotics. The relations among OMPs quantity, permeability, and antibiotics resistance are much better established for $E$. coli than for $P$. aeruginosa, and the possible existence of additional barrier layers besides the outer membrane in the latter species cannot be excluded (Livermore, 1988).

In the present study, a new technique was adopted to selectively solubilize the cytoplasmic membrane with sodium lauroyl sarcosinate for isolation of the outer membrane proteins of P.aeruginosa strains. This method was described by (Zuo et al., 1999) as a relative simple method.

The outer membrane proteins (OMPs) profile analysis of AMC sensitive and resistant strains of $P$. aeruginos $a$ were detected by SDS-PAGE. AMC sensitive $P$. aeruginosa serotype $\mathrm{K}$ and $\mathrm{H}$ induced three common protein bands equal or about (35.713, 26-27 and 23 KD). AMC sensitive $P$. aeruginosa serotype $\mathrm{K}$ additionally induced a protein band of $31.159 \mathrm{KD}$. Whereas AMC resistant $P$. aeruginosa serotype $G$ modified its outer membrane protein patterns. It has two more protein bands of 52.142 and 
Table (1): The results of antibiotic susceptibility test and the presence of $b^{\prime} a_{\mathrm{TEM}}$ gene in the clinical $P$. aeruginosa strains of chicken origin.

\begin{tabular}{|c|c|c|c|c|c|c|}
\hline \multirow{2}{*}{ Antibiotic } & \multicolumn{6}{|c|}{ P. aeruginosa serotypes } \\
\hline & $\mathbf{F}$ & $\mathbf{L}$ & G & $\mathbf{K}$ & $\mathbf{H}$ & $\mathbf{F}$ \\
\hline Amoxicillin-clavulanic acid (AMC, $30 \mu \mathrm{g})$ & $\mathrm{R}$ & $\mathrm{R}$ & $\mathrm{R}$ & $\mathrm{S}$ & $\mathrm{S}$ & $\mathrm{S}$ \\
\hline Ampicillin (Amp, 10 $\mu \mathrm{g})$ & $\mathrm{R}$ & $\mathrm{R}$ & $\mathrm{R}$ & $\mathrm{R}$ & $\mathrm{S}$ & $\mathrm{S}$ \\
\hline Ceftriaxone (CRO, 30 $\mu \mathrm{g})$ & I & I & I & S & $\mathrm{S}$ & $\mathrm{S}$ \\
\hline Neomycin (N, 30ug) & I & I & $\mathrm{R}$ & $\mathrm{R}$ & $\mathrm{R}$ & $\mathrm{S}$ \\
\hline Streptomycin (S, 10ug) & I & $\mathrm{S}$ & $\mathrm{S}$ & $\mathrm{S}$ & $\mathrm{S}$ & $\mathrm{S}$ \\
\hline Norfloxacillin (NOR, $10 \mu \mathrm{g}$ ) & S & S & S & $\mathrm{S}$ & $\mathrm{S}$ & $\mathrm{S}$ \\
\hline$B l a_{\mathrm{TEM}}$ gene detected by the PCR & - & - & + & & & \\
\hline
\end{tabular}

Table (2): Characterization of AMC sensitive and resistant $P$. aeruginosa outer membrane protein by SDS- PAGE technique.

\begin{tabular}{|c|c|c|c|c|c|c|c|c|}
\hline \multirow[b]{3}{*}{ Band } & \multirow{2}{*}{\multicolumn{2}{|c|}{ Marker }} & \multicolumn{6}{|c|}{ The OMPS of $P$. aeruginosa serotype } \\
\hline & & & \multicolumn{2}{|c|}{$* \mathbf{K}$} & \multicolumn{2}{|c|}{$* \mathbf{H}$} & \multicolumn{2}{|c|}{$* \mathbf{G}$} \\
\hline & Mol. W. & $\%$ & Mol. W. & $\%$ & Mol. W. & $\%$ & Mol. W. & $\%$ \\
\hline 1 & 97 & 18.2 & 35.713 & 20.9 & 35.713 & 4.91 & 52.142 & 15.4 \\
\hline 2 & 66 & 15.3 & 31.159 & 12.2 & 27.164 & 6.20 & 38.525 & 9.78 \\
\hline 3 & 45 & 17.1 & 26.107 & 16.2 & 23.174 & 88.9 & 30.690 & 7.12 \\
\hline 4 & 30 & 13.2 & 22.869 & 50.7 & & & 27.164 & 5.58 \\
\hline 5 & 20.1 & 23.3 & & & & & 22.569 & 62.1 \\
\hline 6 & 14.2 & 13.0 & & & & & & \\
\hline
\end{tabular}

The molecular weight is in KD and the amount of the OMPs band is in percentage. The OMPs were loaded ir of approximately $35 \mathrm{mg} * \mathrm{~K}$ is the AMC sensitive $P$. aeruginosa serotype $\mathrm{K}$. ${ }^{*} \mathrm{H}$ is the AMC sensitive $P$. $a_{1}$ serotype $\mathrm{H}$. ${ }^{*} \mathrm{G}$ is the $\mathrm{AMC}$ resistant $P$. aeruginosa serotype $\mathrm{G}$ and positive for $b l a_{\mathrm{TEM}}$ gene.

Table (3): The relationship between the level of the anti-outer membrane protein antibodies detected by ELISA, the OMPs bands detected by SDS-PAG and the AMC susceptibility of $P$. aeruginosa.

\begin{tabular}{lccc}
\hline & \multicolumn{3}{c}{ OMPs of $\boldsymbol{P}$. aeruginosa serotype } \\
& K & H & G \\
\hline The mean OD $(\mathbf{4 0 5} \mathbf{~} \mathbf{m})$ of sera of the injected mice grot & 0.627 & 0.562 & 0.542 \\
The mean OD $(\mathbf{4 0 5} \mathbf{~} \mathbf{m})$ of the control group & 0.174 & 0.169 & 0.158 \\
The AMC sensitivity & $\mathrm{S}$ & $\mathrm{S}$ & $\mathrm{R}$ \\
The number of bands detected by SDS -PAGE & 4 & 3 & 5 \\
\hline
\end{tabular}


38.525 KD than the AMC sensitive $P$. aeruginosa. Three shared protein bands were shown between AMC sensitive and AMC resistant $P$. aeruginosa about $(31,27$ and 23 KD). Hostacka and Karelova, (1997) revealed protein bands of 45, 38, 35 and $23 \mathrm{KD}$ from control $P$. aeruginosa strains. While Zhang et al. (2000) stated that many of the MDR strains of $P$. aeruginosa expressed either moderate or high levels of a novel outer membrane protein band of about $50 \mathrm{kD}$ molecular mass. It can therefore be safely stated that sodium dodecyl sulfate-polyacrylamide gel electrophoresis of OMPs from AMCr strains of $P$. aeruginosa (resistant to AMC) revealed a unique OMP of $52.142 \mathrm{KD}$ when compared with AMCsusceptible strains of $P$. aeruginosa. This unique OMP of $52.142 \mathrm{KD}$ is involved in AMC resistance specifically. These suggestions are similar to those previously reported by (Livermore, 1988) who supported that the outer membrane, composed of porin proteins, which is the major individual barrier. He added that the outer membrane proteins of $P$. aeruginosa play important though not exclusive role in enhancing strain resistance against the AMC. Furthermore, Okamoto et al., (2001) confirmed that, resistance mechanism in $P$. aeruginosa was not strong after loss of either the outer membrane barrier.

It is interesting to note from our results that the OMPs overproduction of AMC resistant $P$. aeruginosa is associated with cross resistance to other antibiotic such as ampicillin, ceftriaxone and neomycin. The finding agree with that of (Masuda et al., 1995) who reported that outer membrane proteins responsible for multiple drug resistance in $P$. aeruginosa induced a band of approximately $50 \mathrm{KD}$.

Although $P$. aeruginosa has an outer membrane with low permeability (Yoshimura and Nikaido, 1982) that can enhance strain resistance against some antibiotics (Cipriani et al., 1995). This alone does not adequately explain $P$. aeruginosa intrinsic resistance, as an additional mechanism interfering with the access of the agents to their targets in this bacterium (Nikaido, 1985). It was clear from the obtained results in this work that the expression of bla TEM $_{\text {Tene in the AMC resistant }}$ $P$. aeruginosa strains indicated that the outer membrane proteins acting in synergy with betalactamase encoded by $b l a_{\mathrm{TEM}}$ gene. This synergistic effect probably responsible for AMC resistance in $P$. aruginosa. The same was obtained by Briñas et al. (2002) who added that susceptibility to $\beta$-lactamase inhibitors could be affected by different mechanisms. The most frequent one is the hyperproduction of classical $\beta$-lactamases or the synthesis of inhibitorresistant TEM (IRT) $\beta$-lactamases by amino acid substitutions in TEM-1 or TEM-2 that encoded by bla $a_{\text {TEM }}$ gene or by other possible changes in membrane quantity and permeability.

The outer membrane proteins can influence the virulence and immunological properties of bacteria (Hale et al., 1983). Attention has been addressed to the role of the outer membrane proteins of $P$. aeruginosa in the induction of specific immunity (Grundmann et al., 1991 and Jang et al., 1999). Our research has been focused on the antibodies against the outer membrane proteins from AMC susceptible and resistance strains. Alexander and Fisher (1974) and Gilleland et al., (1984) successfully used purified outer membrane proteins of Pseudomonas aeruginosa to induce OMPsspecific IgG antibodies in sera of mice.

In the present study specific antibody against two doses of $50 \mu \mathrm{g}$ of OMPs of sensitive or bla $_{T E M}$ positive AMC resistant $P$. aeruginosa strains were determined by ELISA. Analysis of OD values of the pooled sera of mice, two weeks after last $\mathrm{S} / \mathrm{C}$ injection indicated no immunogenic differences between antibodies afforded by OMPs of AMC susceptible strains of $P$. aeruginosa or resistant with $b l a_{T E M}$ gene.

It is important to note that there was no relation between numbers of bands detected by SDS-PAGE and the levels of anti-outer membrane protein antibodies detected by ELISA. These findings suggested that the additional outer membrane protein band of $52.142 \mathrm{kD}$ does not affect the immubogenic responce. Norris and Sciortino (1988) produced monoclonal antibodies against an OMP band specific for the resistant strains of $P$. aeruginosa. Furthermore, Caballero et al. (2001) found a correlation between the levels of antibodies and number of bands of the OMPs of $P$. aeruginosa strains of human origin.

In conclusion, resistance to amoxicillin clavulanic acid in $P$. aeruginosa strains could depend not only, the present of $b l a_{\mathrm{TEM}}$ gene but also on outer membrane protein alterations. There was no association between immunogenicity of the outer membrane proteins and resistance of $P$. aeruginosa. 


\section{References}

Alexander, J. W. and Fisher, M. W. (1974): Immunization against Pseudomonas infection after thermal injury. J. Infect. Dis., 130: 152-158.

Ball, A. P.; Geddes, A. M.; Davey, P.; Farrell, L. D. and G. R. Brookes, (1980): Clavulanic acid and amoxicillin: a clinical bacteriological and pharmacological study. Lancet 1: 620-1623.

Belaaouaj, A., C.; Lapoumeroulie, M. M.; Caniça, G.; Vedel, P.; Névot, R.; Krishnamoorthy, and Paul, G. (1994): Nucleotide sequences of the genes coding for the TEM-like $\beta$-lactamases IRT-1 and IRT-2 (formerly called TRI-1 and TRI-2). FEMS Microbiol. Lett., 120: 75-80.

Briñas, L.; Zarazaga, M.; Sáenz, Y.; Ruiz-Larrea F. and Torres, C. (2002): $\beta$-Lactamases in ampicillinresistant Escherichia coli isolates from foods, humans, and healthy animals. Antimicrob. Agents Chem., 46: 31563163.

Caballero, E.; Drobnic, M. E.; Perez, M. T.; Manresa, J. M.; Ferrer, A. and Orriols, R. (2001): AntiPseudomonas aeruginosa antibody detection in patients with bronchiestasis without cystic fibrosis. Thorax, 56: 669-674.

Cullmann, W.; Buscher, K. H. and Opferkuch, W. (1986): Characterization of imipenem resistance in Pseudomonas aeruginosa: decreased phenotypic expression of outer membrane proteins D1 and D2. Immun. Infekt., 14 (6): 227-229.

Cipriani, P.; Giordano, A.; Magni, A.; Papa, F.; and Filadoro, F. (1995): Outer membrane alterations in Pseudomonas aeruginosa after five-day exposure to quinolones and carbapenems. Drugs Exp., Clin., Res., 21: 139-144.

Gilleland, H. E.; Parker, M. G.; Matthews, J. M.; and Berg, R. D. (1984): Use of purified outer membrane protein F (porin) of Pseudomonas aeruginosa as a protective vaccine in mice. Infect. Immun., 44: 49-54.

Grundmann, T.; Freihorst, J.; Kubesch, P.; Tummler, B.; von der Hardt, H. (1991): Local immunization against Pseudomonas aeruginosa with an outermembrane-protein vaccine. Pneumologie., 45 (11): 924927.

Hale, T. L.; Sansonetti, P. J.; Schad, P. A.; Austin, S. and Formal, S. B. (1983): Characterization of virus plasmids and plasmid-associated outer membrane proteins in Shigella flexneri, Shigella sonnei and Escherichia coli. Infect. Immun., 40: 340 -350

Hames, B. D. and Rickwood, D. (1987): Gel electrophoresis of proteins. A practical approach. $6^{\text {th }}$ ed., IRL press,Oxford.Washington.

Hostacka, A. and Karelova, E. (1997): Outer membrane proteins profiles of Pseudomonas aeruginosa after the post-antibiotic effect of imipenem. Microbios., 90 (362): 45-50.

Jang, I. J.; Kim, I. S.; Park, W. J.; Yoo, K. S.; Yim, D. S.; Kim, H. K.; Shin, S. G.; Chang, W. H.; Lee, N. G.; Jung, S. B.; Ahn, D. H.; Cho, Y. J.; Ahn, B. Y.; Lee, Y.; Kim, Y. G.; Nam, S. W. and Kim, H.S. (1999): Human immune response to a Pseudomonas aeruginosa outer membrane protein vaccine. Vaccine, 17 (2): 158-68.

Kaye, K. S.; Howard, S. G.; Mitchell, J. S.; Lata, V.; Youlin, Q.; Paola, C. D.; Matthew, H. S.; Greg, A.; Rasheed, J. K.; and Fred, C. T. (2004): Variety of $\beta$ Lactamases Produced by Amoxicillin-Clavulanate-
Resistant Escherichia coli Isolated in the Northeastern United States. Antimicrob. Agents Chem., 48: 1520-1525.

Laemmli, U. K. (1970): Cleavage of structural proteins during the assembly of the head of bacteriophage T4. Nature, 227: 680-685.

Leitner, G.; Melamed, D.; Drabkin, N.; Heller, E.D. (1990): An enzyme-linked immunosorbent assay for detection of antibodies against Escherichia coli: association between indirect hemagglutination test and survival. Avian Dis., 34: 58-62.

Li, X. Z.; Zhang, L. and Poole, K. (2000): Interplay between the MexA-MexB-OprM multidrug efflux system and the outer membrane barrier in the multiple antibiotic resistance of Pseudomonas aeruginosa. J. Antimicrob. Chem., 45: 433-436.

Livermore, D. M. (1988): Permeation of beta-lactam antibiotics into Escherichia coli, Pseudomonas aeruginosa, and other gram negative bacteria. Rev. Infect. Dis., 10: 691-698.

Lowry, O. H.; Rosenbrough, N. J.; Farr, A. L. and Randall, R. J. (1951): Protein measurement with the folin phenol reagent. J. Biol. Chem., 193: 265-275.

Masuda, N.; Sakagawa, E.; and Ohya, S. (1995): Outer membrane proteins responsible for multiple drug resistance in Pseudomonas aeruginosa. Antimicrob. Agents Chemother., 39: 645-649.

Muthukumar, S. and Muthukkaruppan, V. R. (1993): Mechanism of protective immunity induced by porinlipopolysaccharide against murine salmonellosis. Infect. Immun., 61: 3017-3025.

National Committee for Clinical Laboratory Standards (1993): Methods for antimicrobial susceptibility testing of aerobic bacteria. $2^{\text {nd }}$ ed. Approved standard M7-A3., Villanova, $\mathrm{Pa}$.

Nikaido, H. (1985): Role of permeability barriers in

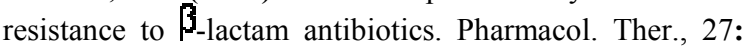
197-231.

Norris, S. A. and Sciortino C. V. (1988): Monoclonal antibody to an aminoglycoside-resistance factor from Pseudomonas aeruginosa. J. Infect. Dis., 158 (6): 132413248.

Okamoto, K.; Gotoh, N. and Nishino, T. (2001): Pseudomonas aeruginosa reveals high intrinsic resistance to penem antibiotics: Penem resistance mechanisms and their interplay. Antimicrob. Agents and Chem., 45: 19641971.

Putman, M.; Hendrik, W. V. and Konin, W. N. (2000): Molecular properties of bacterial multidrug transporters. Microbiol. Mol. Biol. Rev., 64: 672- 693.

Quinn, P. J.; Markey, B. K.; Carter, M. E.; Ponnelly, W. J.; Leonard, F. C.; Maguire, D. (2002): Veterinary Microbiology and Microbial diseases. $2^{\text {nd }}$ ed., Blackwell Sci., USA.

Yoshimura, F., and Nikaido, H. (1982): Permeability of Pseudomonas aeruginosa outer membrane to hydrophilic solutes. J. Bacteriol., 152: 636-642.

Zhang, L.; Xian-Zhi, L. and Keith, P. (2000): Multiple Antibiotic Resistance in Stenotrophomonas maltophilia: Involvement of a Multi-drug Efflux System. Antimicrob. Agents and Chemother., 44 (2): 287-293.

Zuo, L.; Zhou, J. and Yao, T. (1999): Purification of outer membrane proteins in Pseudomonas aeruginosa by high performance ion-exchange liquid chromatography. Se. Pu., 17: 46-48. 